\title{
Design of optical systems with extended depth of field: an educational approach to wavefront coding techniques
}

\author{
Carme Ferran, Salvador Bosch, and Artur Carnicer
}

\begin{abstract}
A practical activity designed to introduce wavefront coding techniques as a method to extend the depth of field in optical systems is presented. The activity is suitable for advanced undergraduate students since it combines different topics in optical engineering such as optical system design, aberration theory, Fourier optics, and digital image processing. The paper provides theoretical background and technical information for performing the experiment. The proposed activity requires students able to develop a wide range of skills, since they are expected to deal with optical components, including spatial light modulators, and develop scripts to perform some calculations.
\end{abstract}

Index Terms

Education, Image processing, Optical imaging, Optical transfer function, Spatial light modulators

\section{INTRODUCTION}

DEPTH-OF-FIELD (DOF), the distance range over which an object remains in-focus for a fixed image detection plane, is one of the key parameters for an optical engineer when designing

This paper has been partially supported by the CICYT (the Spanish R+D Agency) project DPI2008-04175 and the Institute of Education Sciences (UB) grant REDICE10 1001-05.

C. Ferran, S. Bosch, and A. Carnicer are with the Applied Physics and Optics Department, Universitat de Barcelona, Barcelona, Spain e-mail: artur.carnicer@ub.edu

C. Ferran thanks a BRD grant from the Universitat de Barcelona. 
an optical instrument. The trivial method for increasing DOF is to reduce the aperture of the instrument; in this way, the system presents less aberration, which results in better image quality. Nevertheless, a smaller aperture (i) increases the role of diffraction, which limits the resolving power of optical instruments, and (ii) reduces the amount of light that can be gathered by the system. Wavefront coding (WFC) is a state-of-the-art technique that enables DOF improvement without jeopardizing other design parameters such as the luminosity of the instrument. WFC was first proposed by Dowsky and Cathey [1], [2] and nowadays is widely used in the design of optical setups [3]-[5]. The technique is based on the modification of the wavefront (or wavefront coding, using Dowsky and Cathey words) by means of a suitable phase mask (PM) placed at the aperture stop of the system. The aim of WFC is to modify the Point Spread Function (PSF) in such a way that it becomes invariant over a range of distances around the image plane. However, the coded images look blurred and they must be digitally filtered in order to remove the coding of the PSF; finally, an image with diffraction-limited quality (ie, as obtained with a non aberrated optical system whose only limitation is diffraction) may be obtained.

PMs are usually written on transparent materials using refractive optics techniques. Taking into account that phase delay is proportional to optical path, wavefront coding is achieved by fitting the thickness of the PM to the required phase distribution. Recently, the use of liquid crystal displays (LCD) as spatial light modulators in WFC was proposed [6], [7]. Generally speaking, LCDs are pixelated displays controlled by a computer. LCDs modulate both the amplitude and phase of the beam and are used to encode information in digital holography applications. Such displays allow flexible implementation of the PM, since the parameters that control its behavior can be modified dynamically.

A practical exercise to introduce WFC to advanced students (upper undergraduate or master's degree) in Applied Physics, Optical Engineering, Photonics, Electrical Engineering or other related disciplines is presented. In particular, the practical has been designed having in mind the syllabus of the Image Processing (IP) course at the Universitat de Barcelona. Since WFC is a multidisciplinary field, a basic knowledge of different subjects, such as optical design, including theory of aberrations and Fourier optics, digital image processing, and some programming skills, is required. The objective of the proposed activity is to develop a complete WFC system from scratch with minimal guidance, following the project-based learning approach (see for instance [8]). This requires students able to develop a wide range of skills, since they are expected to deal 
with optical components, LCDs, CCD cameras and to develop scripts to perform calculations. The use of a plurality of techniques is necessary when designing an optical instrument; some authors have pointed out this fact [9], [10]. In summary, the following issues are going to be addressed:

- Setting up an optical system

- Calibration of liquid crystal devices

- Calculation of the spherical aberration of an optical system

- Phase mask design and calculation of computer generated holograms

- Reconstruction of defocused images using the Wiener filter

The detected encoded wavefront has to be decoded using signal processing techniques. However, the study of the characteristics of WFC as a three dimensional communication channel is not addressed in the present work. The interested reader can find out more information about this topic see, for instance [11] and references therein.

The paper is organized as follows: in section II, the structure of the IP course is presented; the theoretical basics required to understand the underlying concepts are reviewed in section III. In section IV the experiment is introduced and in section $\mathrm{V}$, an assessment of the project is discussed. Finally, the conclusions are presented in section VI.

\section{COURSE DESCRIPTION}

The practical is part of the IP course at the Universitat de Barcelona; IP is an optional course for advanced undergraduates in Applied Physics. It comprises of 65 hours, equivalent to 6 ECTScredits (European Credit Transfer and Accumulation System), consisting on 38 lectures and 9 labs. Those who enroll this course have taken compulsory credits in physical optics, programming and, an advanced course in mathematics which includes Fourier analysis. Some of them are also following an elective course in Photonics. Consequently, it is expected that the students possess the required background to follow the course.

On average, about 30 students enroll IP each year and the success rate is approximately 75 $\%$. For details see table I.

The syllabus of IP is divided in two parts: Digital Image Processing (DIP) and Optical Image Formation (OIF). Joining this two topics in the same course is advisable since DIP techniques are used in the design of optical systems to assist and improve its behavior. For instance, defocused 
TABLE I

Number of STUDENTS ENROLLED AND SUCCESS RATE

\begin{tabular}{lrr}
\hline Year & Total students & Successful students \\
\hline 2006 & 32 & $27(84 \%)$ \\
2007 & 30 & $19(63 \%)$ \\
2008 & 20 & $16(80 \%)$ \\
2009 & 37 & $25(68 \%)$ \\
2010 & 35 & $28(80 \%)$ \\
\hline Total & 154 & $115(74 \%)$ \\
\hline
\end{tabular}

images obtained by means of an aberrated optical system can be enhanced using image restoration techniques, provided that the PSF of the optical system is known. Moreover, DIP and OIF share a common framework based on Fourier analysis that allow an unified presentation. Nevertheless, DIP and OIF are often perceived by students as unconnected topics and, consequently one of the goals of this experiment is to demonstrate students how digital and optical techniques are jointly used for designing optical systems.

Tables II and III show the topics covered and the list of proposed labs.

\section{THEORETICAL BACKGROUND}

\section{A. Optical Systems and the Point Spread Function}

From the geometrical point of view, an optical instrument can be sketched by tracing rays through the surfaces that comprise the system, using Snell's law [12]. Moreover, a general imaging device can also be described as a black box consisting of two planes containing the entrance and exit pupils; in particular, the latter is the geometrical projection in image space of the physical limiting aperture of the system. It can be demonstrated [13] that an imaging system can be fully analyzed in terms of signal theory just from the knowledge of the position and radius $R$ of the exit pupil (EP) and the scalar electromagnetic light field in this plane. Let $f_{o}\left(x_{1}, y_{1}\right)$ be the function that describes the intensity of the object to be imaged through the optical system. Assuming a quasi-monochromatic (wavelength $\lambda$ ) incoherent optical system and according to paraxial optics theory, the intensity of the image function is $f_{g}(x, y) \propto f_{o}\left(\frac{x_{1}}{\beta}, \frac{y_{1}}{\beta}\right)$, where $\left(x_{1}, y_{1}\right)$ and $(x, y)$ are the coordinates in the object and image plane; and $\beta$ is the magnification. Figure 
TABLE II

IMAGE PROCESSING SYLLABUS

1) The $2 \mathrm{D}$ Fourier transform

- Review of Fourier transform properties

- The fast Fourier transform algorithm

2) Scalar Diffraction theory:

- The angular spectrum of plane waves framework.

- Fresnel diffraction

- Fraunhofer diffraction

3) Analysis of coherent optical systems.

- Fourier transforming properties of lenses.

- Image formation

- Optical spatial filtering

4) Frequency analysis of optical imaging systems

- Diffraction limited coherent optical systems

- Diffraction limited incoherent optical systems

- Aberrations

5) Image enhancement and filtering

- Image representation

- Intensity transformations.

- Linear and non-linear filtering

- Fourier domain filtering

6) Image restoration and reconstruction

- Inverse filter

- Wiener filter

- Constrained least squares filter

7) Advanced topics: digital holography, spatial light modulators, wavefront coding.

1 depicts such an optical system. In what follows a radially symmetrical optical system is considered.

For a linear and intensity invariant optical system, the intensity pattern $f_{i}(x, y, z)$ in an observation plane at a distance $z$ from the EP can be described as the convolution of the geometrical image, $f_{g}(x, y)$, and the corresponding incoherent PSF, $|h(x, y, z)|^{2}$, i.e.: 
TABLE III

LAB LIST

1) Review of programming techniques in Matlab/Octave

2) Fresnel diffraction: Talbot effect, Fresnel lenses

3) Fourier properties of lenses. Coherent optical filtering

4) Modulation transfer function

5) Image representation and intensity transformation techniques

6) Introduction to Fourier image processing

7) Linear and non-linear-filtering

8) Image restoration and reconstruction

9) Wavefront coding techniques

\section{OBJECT PLANE}

OPTICAL SYSTEM
PARAXIAL IMAGE OBSERVATION

PLANE

PLANE

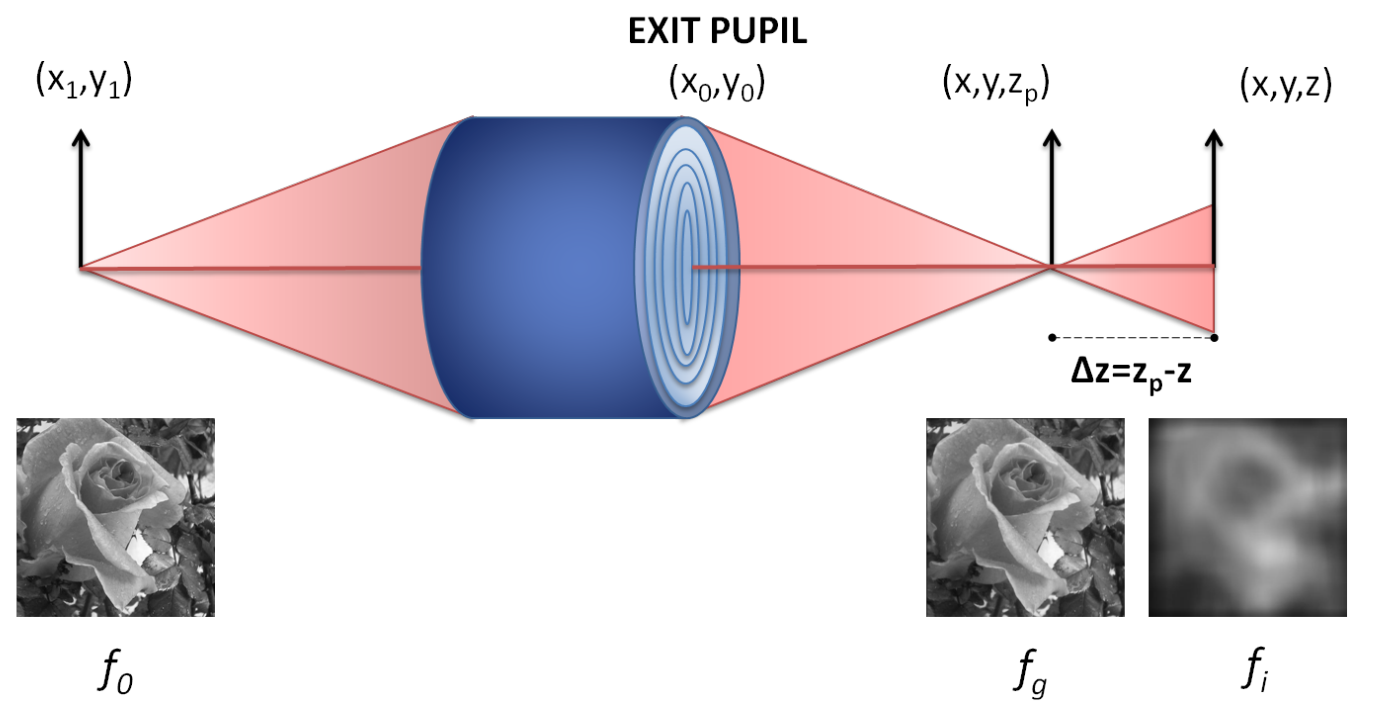

Fig. 1. Generalized (black box) optical system

$$
\begin{gathered}
f_{i}(x, y, z)=\int f_{g}\left(x^{\prime}, y^{\prime}\right)\left|h\left(x-x^{\prime}, y-y^{\prime}, z\right)\right|^{2} d x^{\prime} d y^{\prime} \\
=f_{g}(x, y) *|h(x, y, z)|^{2} .
\end{gathered}
$$


Using the Fresnel diffraction framework, the complex amplitude of the incoherent PSF, $h(x, y, z)$, is calculated using:

$$
\begin{aligned}
& h(x, y, z) \propto \frac{1}{\lambda z} \int_{\mathrm{EP}} \exp \left[i k W\left(x_{0}, y_{0}\right)\right] . \\
& \cdot \exp \left[i k \frac{\Delta z}{2 z^{2}}\left(x_{0}^{2}+y_{0}^{2}\right)\right] \exp \left[i \frac{k}{z}\left(x x_{0}+y y_{0}\right)\right] d x_{0} d y_{0},
\end{aligned}
$$

where $W\left(x_{0}, y_{0}\right)$ is the pupil aberration function that accounts for the effective path length error in the wavefront, i.e. how the wave is distorted when it crosses the optical system; $\left(x_{0}, y_{0}\right)$ are the coordinates in the EP plane normalized to the edge of the pupil and $k=2 \pi / \lambda$ is the wave number. The so-called spherical aberration is dominant at those points that belong to the observation plane and are close to the optical axis: according to the Seidel theory of aberrations, the pupil function can be written as

$$
W\left(x_{0}, y_{0}\right)=A_{s}\left(x_{0}^{2}+y_{0}^{2}\right)^{2}
$$

where $A_{s}$ is a constant that accounts for the severity of this aberration [14], [15]. The second exponential term in Equation 2 provides information on the defocus in the observation plane; here, $\Delta z=z_{p}-z$ is the distance between the paraxial image plane at $z_{p}$ and the observation plane considered to be at $z$. Finally, the last exponential is just the Fourier transform kernel. Notice that the integral must be evaluated within the EP.

Usually, equations are written in terms of the image space variables, even though real imaging systems work for a fixed sensor position and defocus is a result of different object distances (as seen later in Figure 7). For a given amount of defocus ${ }^{1}, \Delta z$ can easily be translated into object space by means of the thin lens equation [12]

$$
\frac{1}{s}+\frac{1}{s^{\prime}}=\frac{1}{f^{\prime}}
$$

where $s$ is the distance from the object to the front nodal point and $s^{\prime}$ the distance from the back nodal point to the paraxial image.

\footnotetext{
${ }^{1}$ Defocus is commonly expressed as $A_{d}=\Delta z / 8 \lambda F_{\#}^{2}$ in $\lambda$ units, where $F_{\#}=\frac{f}{\phi_{E P}}$, with $f$ the focal distance and $\phi_{E P}$ the exit pupil diameter. This value can be defined in either the object or in the image plane depending on the $\Delta z$ and $F_{\#}$ specification.
} 


\section{B. Wavefront Coding}

WFC consists of the addition of a PM, whose mathematical expression is $\exp \left(i k P\left(x_{0}, y_{0}\right)\right)$, to the EP of the optical system. To extend DOF, several phase distributions have been proposed; [1], [2], [16]. The cubic plate is the most common and widely used:

$$
P\left(x_{0}, y_{0}\right)=\alpha\left(x_{0}^{3}+y_{0}^{3}\right)
$$

where $\alpha$ is the strength of the plate, measured in lambda units. Very often, the PM is designed in such a way that the effect of spherical aberration is compensated for, i.e.

$$
P\left(x_{0}, y_{0}\right)=\alpha\left(x_{0}^{3}+y_{0}^{3}\right)-A_{s}\left(x_{0}^{2}+y_{0}^{2}\right)^{2} .
$$

Rewriting Equation 2, the complex-amplitude of the PSF of a wavefront-coded aberration-free optical system becomes:

$$
\begin{aligned}
& h_{\mathrm{wfc}}(x, y, z) \propto \frac{1}{\lambda z} \int_{\mathrm{EP}} \exp \left(i k \alpha\left(x_{0}^{3}+y_{0}^{3}\right)\right) \cdot \\
& \cdot \exp \left(i k \frac{\Delta z}{2 z^{2}}\left(x_{0}^{2}+y_{0}^{2}\right)\right) \cdot \\
& \cdot \exp \left(\frac{i k}{z}\left(x x_{0}+y y_{0}\right)\right) d x_{0} d y_{0} .
\end{aligned}
$$

The resulting PSF, $\left|h_{\mathrm{wfc}}\right|^{2}$, is approximately invariant for a certain range of distances around the image plane and, consequently, the resulting images are nearly indistinguishable regardless of the value of $z$ (see references [1], [2]). However, coded images are of low quality and digital restoration is required to remove the degradation introduced by the coded PSF. Using equation 1, the coded image, $f_{c}(x, y, z)$, recorded by a video-camera can be written as:

$$
f_{c}(x, y, z)=f_{g}(x, y) *\left|h_{\mathrm{wfc}}(x, y, z)\right|^{2} .
$$

The goal is to recover the diffraction-limited image, $f_{g}(x, y)$. This image restoration process is easily achieved using the parametric Wiener filter (see for instance [17]). This filter is defined as:

$$
W(u, v)=\frac{H_{\mathrm{wfc}}^{*}(u, v)}{\left|H_{\mathrm{wfc}}(u, v)\right|^{2}+C},
$$




\begin{tabular}{ll}
\hline Lens focal length & $f^{\prime}=208 \mathrm{~mm}$ \\
\hline Object distance & $593 \mathrm{~mm}$ \\
\hline Image distance & $316 \mathrm{~mm}$ \\
\hline Spherical aberration & $A_{s}=26 \lambda$ \\
\hline Cubic phase plate strength & $\alpha=10 \lambda$ \\
\hline Lens diameter & $\phi=60 \mathrm{~mm}$ \\
\hline Lens first surface radius & $R_{1}=59.8 \mathrm{~mm}$ \\
\hline Lens second surface radius & $R_{2}=130.7 \mathrm{~mm}$ \\
\hline Lens refractive index & $n=1.515$ \\
\hline$F_{\#}$ in image plane & $F_{\#}=9.4$ \\
\hline Exit Pupil Diameter & $\phi_{E P}=34 \mathrm{~mm}$ \\
\hline Wavelength & $\lambda=633 \mathrm{~nm}$ \\
\hline
\end{tabular}

TABLE IV

OPTICAL TEST SYSTEM SPECIFICATIONS

where $H_{\mathrm{wfc}}$ is the Fourier transform of $\left|h_{\mathrm{wfc}}\right|^{2}$ and $C$ a constant to be interactively adjusted to provide a good visual result. The restored diffraction-limited quality image is obtained by calculating:

$$
\mathcal{F}^{-1}\left[F_{c}(u, v) \cdot W(u, v)\right]
$$

where $F_{c}(u, v)$ is the Fourier transform of $f_{c}(x, y, z)$; and $\mathcal{F} \mathcal{T}^{-1}$ stands for the inverse Fourier transform operator. It is worth mentioning here that the $H_{\mathrm{wfc}}$ distribution is so designed that it does not present zeros and consequently, $W(u, v)$ is well defined throughout its whole domain.

A set of simulations has been prepared to illustrate the behavior of the preceding equations. The defocus invariant imaging system considered is a single meniscus lens and a cubic phase plate with the nominal values presented in Table IV. Figure $2 \mathrm{a}$ and Figure $2 \mathrm{~b}$ depict a cubic PM (Equation 5) and the same PM with compensation for spherical aberration (Equation 6) as displayed on the light modulator. The corresponding PSF $\left|h_{\mathrm{wfc}}\right|^{2}$ (Equation 7) is presented in Figure 2c.

Figure 3 shows the simulation of the recorded images at $\Delta z=0 \mathrm{~mm}$ (referred to as the best quality image plane $\left.{ }^{2}\right), \Delta z=-5 \mathrm{~mm}$ and $\Delta z=5 \mathrm{~mm}$. The first row corresponds to an

\footnotetext{
${ }^{2}$ The position of the best quality image plane varies depending on the presence of aberrations in the system. Once aberrations are compensated for, the best quality image plane is the paraxial plane.
} 

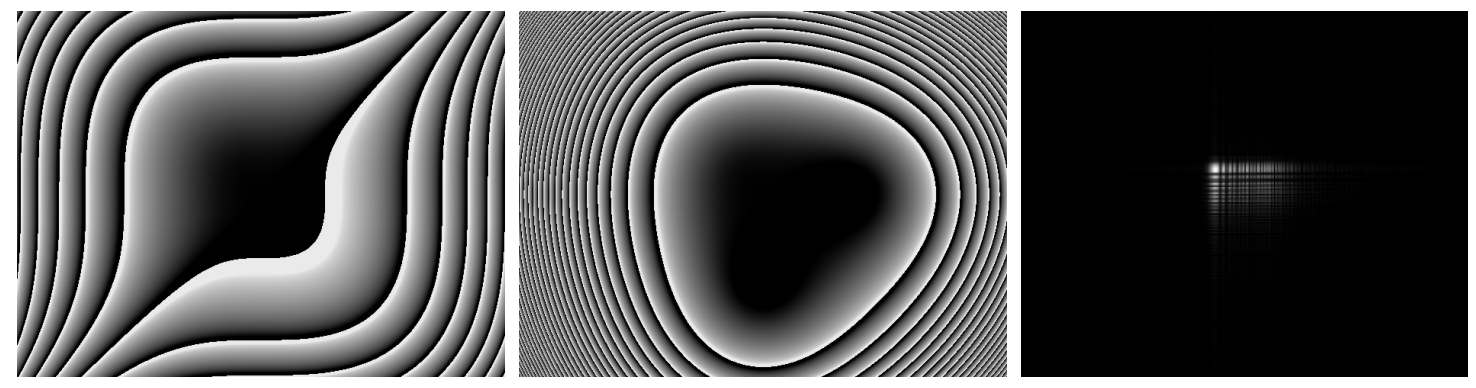

Fig. 2. a) Phase mask $P\left(x_{0}, y_{0}\right), \alpha=10 \lambda, A_{s}=0 \lambda$, b) Phase mask $P\left(x_{0}, y_{0}\right), \alpha=10 \lambda, A_{s}=26 \lambda$, c) Defocus-invariant coded PSF $\left|h_{\mathrm{wfc}}\right|^{2}$

image formation process using a conventional optical system. The image obtained at $\Delta z=0$ $\mathrm{mm}$ is affected by spherical aberration, so the image quality is not very high; images acquired at $\Delta z=-5 \mathrm{~mm}$ and $\Delta z=5 \mathrm{~mm}$ are also affected by defocussing. The second row illustrates how compensation for spherical aberration affects the final image. Here, the imaging system becomes a perfect lens and consequently, a diffraction-limited quality image is obtained at $\Delta z=0 \mathrm{~mm}$. Notice that tolerance to defocusing is lower and the images acquired at $\Delta z=-5 \mathrm{~mm}$ or $\Delta z=5 \mathrm{~mm}$ are of even poorer quality than those obtained when the system is affected by spherical aberration. In row three, a cubic phase plate has been added to the aberration-free lens. The quality of the resulting images is very low but, on the other hand, they are hardly distinguishable. These pictures are affected by the wavefront-coded PSF, $\left|h_{\mathrm{wfc}}\right|^{2}$; to remove the degradation introduced by this PSF, a Wiener filter-based deconvolution is needed. The result of the reconstruction process is shown in the last row: the optical system becomes invariant to defocusing with nearly diffraction-limited quality images.

For a more fundamental analysis of WFC, the interested reader can access references [2] and [18].

\section{IMPLEMENTATION}

In this section, information on how to implement a WFC optical system for teaching purposes is provided. This requires successfully completing several tasks: the first consists of setting up the LCD; then, it is necessary to evaluate the spherical aberration $A_{s}$ introduced by the lens. Once this parameter is known, an appropriate strength $\alpha$ should be selected in order to calculate 


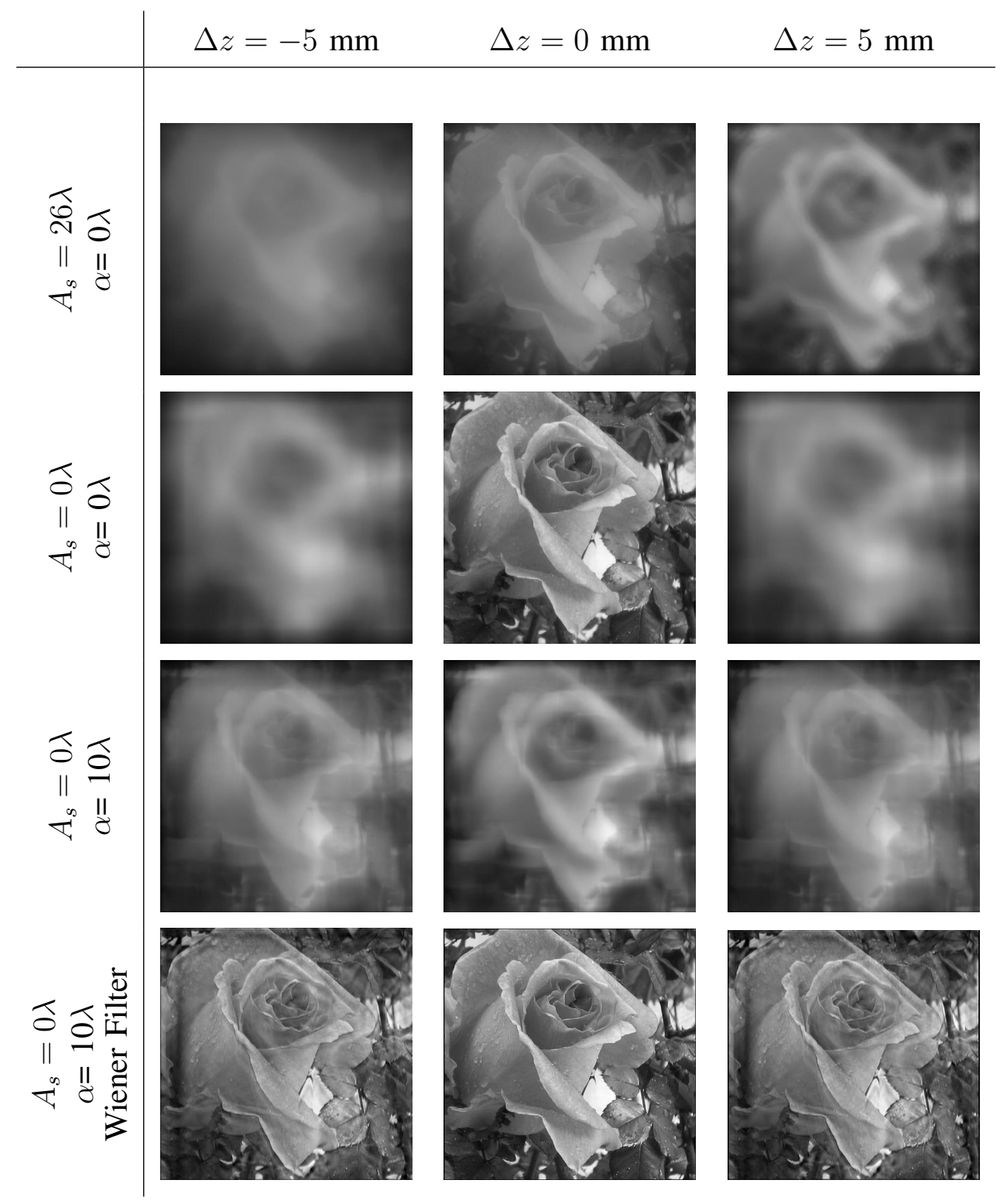

Fig. 3. Simulation of the image formation process through an optical system. Images recorded at $\Delta z=0 \mathrm{~mm}, \Delta z=-5 \mathrm{~mm}$ and $\Delta z=5 \mathrm{~mm}$. First row: conventional optical system. Second row: Aberration-free (perfect) lens. Third row: perfect lens + cubic phase plate. Last row: Wiener filter reconstructed images.

the PM. The next step consists of building the optical setup and finally, a script implementing the Wiener filter has to be prepared ${ }^{3}$.

${ }^{3}$ The reader can access the computing materials described in this section, including MATLAB ${ }^{\circledR}$ scripts, an LCD configuration file and a Labview program that can be used to control the optoelectronic elements of the system. These files can be accessed at http://www.ub.edu/waveng/wfc_practical. 


\section{A. Setting up the LCD}

As pointed out in section I, one possible way to implement WFC phase plates within an optical system is by using an LCD [6]. LCDs are programmable electro-optical devices that can modulate light depending on the applied voltage and the polarization state of the incident light. The behavior of an LCD can be represented by an operating curve, which gives information on the way the device modulates light depending on the gray level assigned to each pixel. Since the aim is to implement phase distributions, the phase-mostly configuration is the most suitable. In the experiment, an LCD panel removed from a commercial Epson video-projector model EMP3000 is used ${ }^{4}$. This panel displays 640x480 pixels with a pixel pitch of $42 \mu \mathrm{m}$ and an 8-bit pixel depth. The phase-mostly configuration is achieved when the polarization direction with respect to the vertical of the laboratory is $-29^{\circ}$ and the polarization direction of the second polarizer is set to $88^{\circ}$. More information about the behavior of the Epson LCD can be found in [20]. Figure 4 shows the dependence of the gray level to be displayed as a function of the calculated phase on a particular pixel. Notice that this particular device cannot modulate phase values between $1.6 \pi$ and $2 \pi$. This drawback can be avoided using the saturation encoding technique proposed in [21] to compensate the diffraction efficiency loss.

\section{B. Calculation of the spherical aberration coefficient}

Spherical aberration is due to an increase in the refraction of rays near the edge of a lens compared to those near the center. In order to compensate for this aberration, the value of the Seidel coefficient $A_{s}$ has to be known. Some commercial programs used in optical system design, such as $\mathrm{ZEMAX}^{5}$, can be used to calculate it. If such software is not available, a computer script is provided (seidelsum.m).

The calculation of the Seidel coefficient is straightforward but is beyond the scope of this paper; however, for the sake of completeness some guidelines to illustrate how this value is obtained are included. The interested reader can look up an optical system design manual such as [22] to expand the information contained here. The algorithm is based on paraxial ray tracing of the marginal ray through all the refractive surfaces of the system. The marginal ray is the ray

\footnotetext{
${ }^{4}$ Some SLM manufacturers provide kits for educational purposes. See, for instance, reference [19]

${ }^{5}$ ZEMAX is a registered trademark of ZEMAX Development Corporation
} 


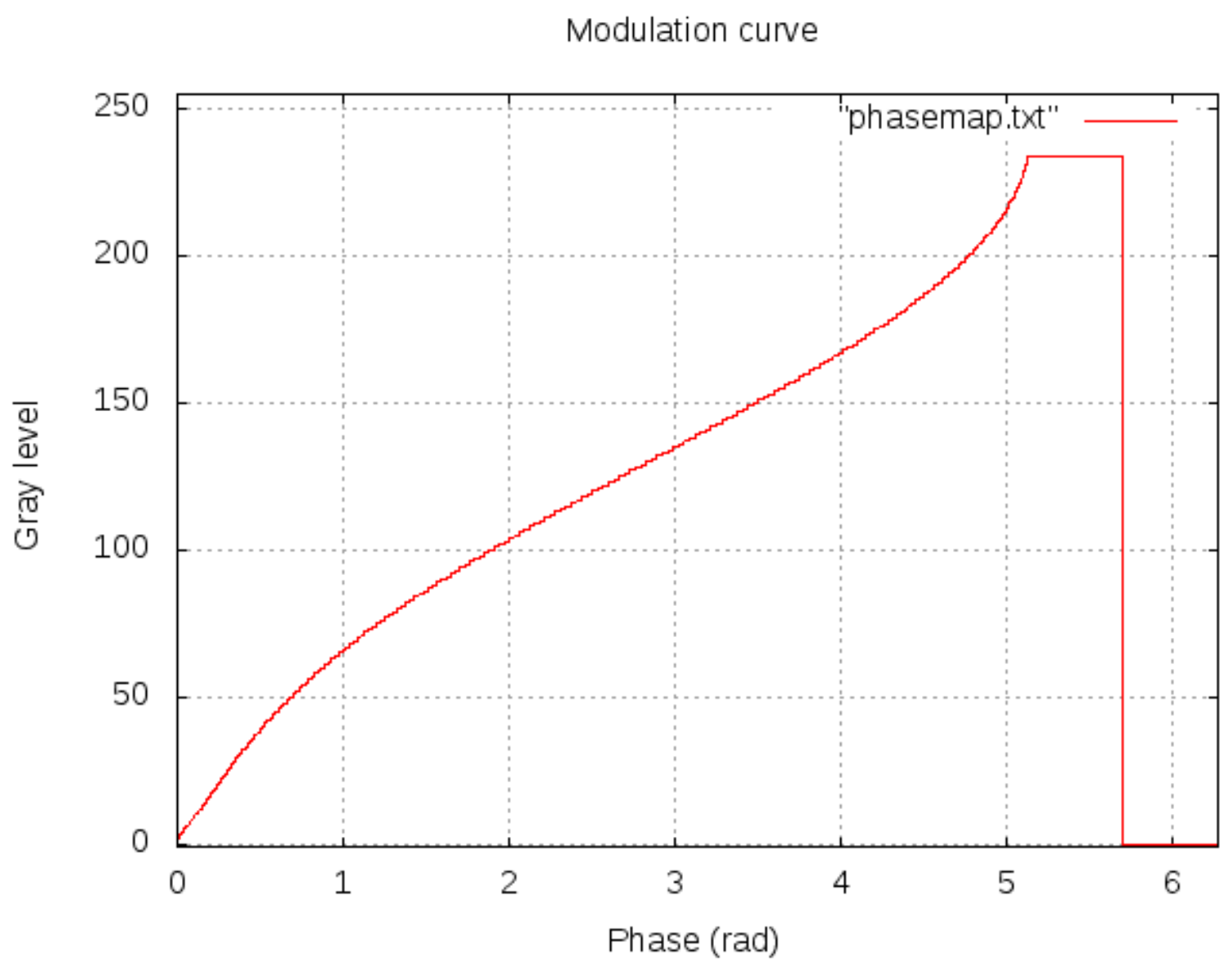

Fig. 4. Epson EMP-3000 phase-mostly operating curve.

that passes from the center of the object through the edge of the aperture stop of the system. To perform this calculation, it is necessary to know the geometrical variables of the optical system (position and radii of the surfaces), refractive indexes and the position of the object and the image planes. The Seidel coefficient, $A_{s}$, for an optical system is given by:

$$
A_{s}=-\frac{1}{8} \sum_{j=2}^{m} A_{j}^{2} h_{j}\left(\frac{u_{j}}{n_{j}}-\frac{u_{j-1}}{n_{j-1}}\right),
$$

where $m$ is the number of surfaces ( $m=1$ is the object plane), $A_{j}$ is the refractive invariant $\left(n_{j} \theta_{j}\right.$, where $\theta_{j}$ is the angle of incidence), $h_{j}$ is the height of the marginal ray on the surface $j, u_{j-1}$ and $u_{j}$ are the incoming and outgoing convergence angles, and $n_{j-1}$ and $n_{j}$, the corresponding values of the refractive index. Figure 5 traces the meridional plane ray between the object plane and the first refractive surface. 
$\mathrm{n}=$ refractive index

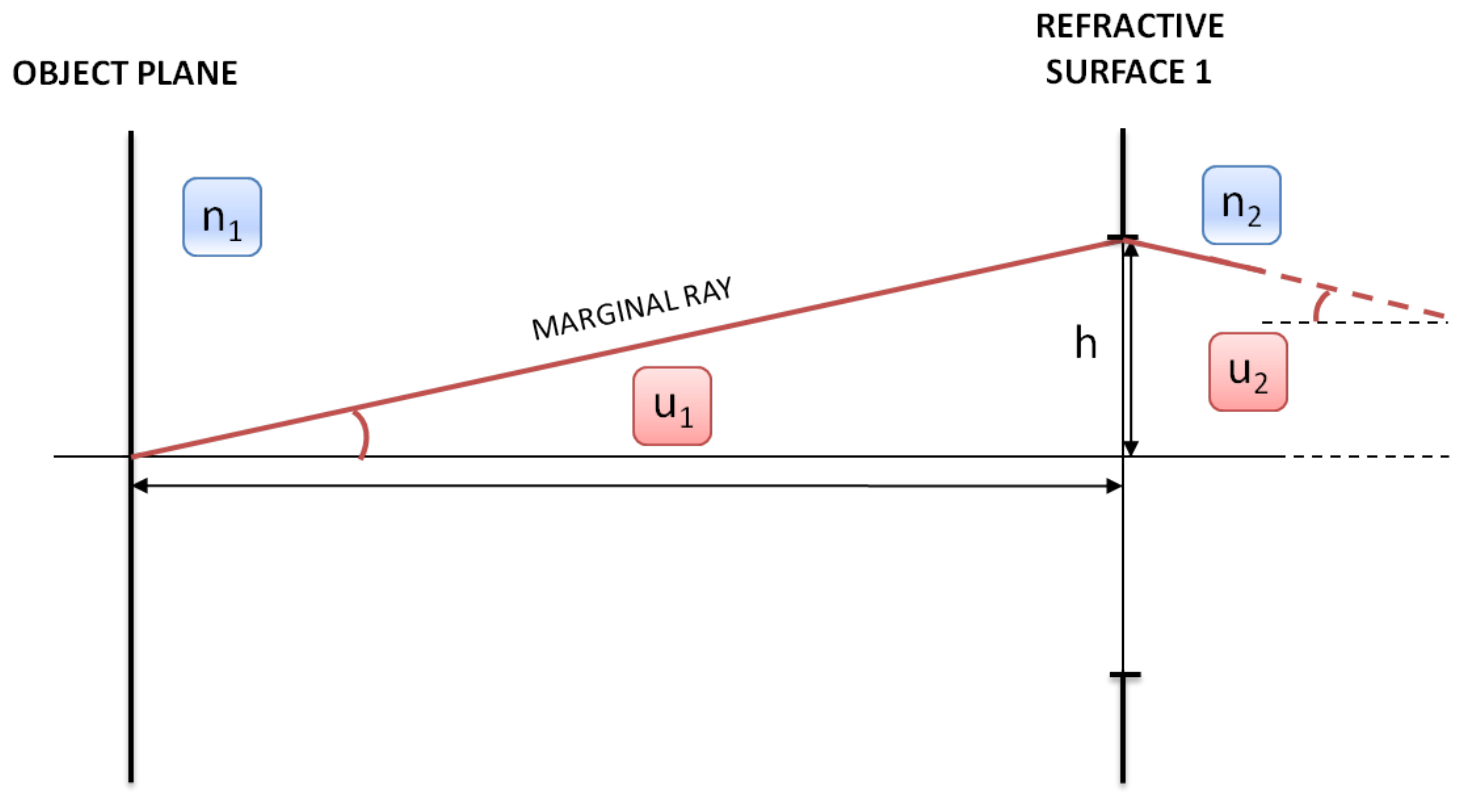

Fig. 5. Marginal ray traced between the first two surfaces of the optical system.

\section{Design of the phase mask}

The PM is calculated by means of Equation 6. This distribution includes the WFC term plus a second term that compensates for spherical aberration; the goal is to obtain an 8-bit image that can be displayed on the LCD. The steps for generating the required PM are:

1) Allocate an $N \times M$ floating point matrix, with $N$ and $M$ the number of rows and columns of the LCD. Notice that the radius of the EP is normalized to unity, and values $x_{0}$ and $y_{0}$ are scaled accordingly.

2) Using Equation 6 prepare the distribution $P\left(x_{0}, y_{0}\right) \bmod 2 \pi$.

3) Generate an 8-bit image using the relationship between the phase modulation and the gray level displayed, and save this bitmap.

In the experiment, the resolution of the LCD is $N \times M=480 \times 640$ pixels, the spherical aberration of the lens under working conditions is $A_{s}=26 \lambda$, and the diameter of the EP is the value of the diagonal of the LCD. The information required to transform the calculated phase $P\left(x_{0}, y_{0}\right) \bmod 2 \pi$ into a gray-level image (Figure 4$)$ is contained in the file phasemap.txt. Figure 6 shows two masks generated with the script createmask.m; the first one corrects a spherical 
aberration of $A_{s}=26 \lambda$ whereas the second one compensates the aberration and also codes the wavefront with a strength of $\alpha=10 \lambda$.
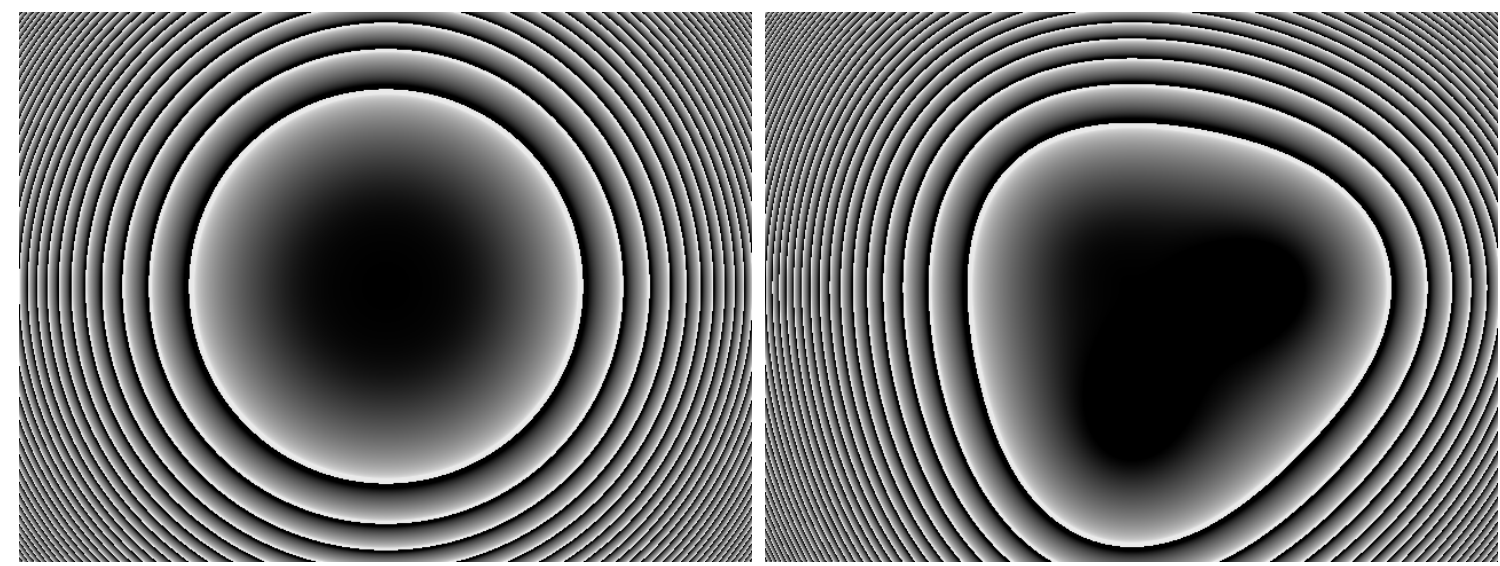

Fig. 6. Phase masks: (a) $A_{s}=26 \lambda, \alpha=0 \lambda$ (b) $A_{s}=26 \lambda, \alpha=10 \lambda$

\section{Wavefront Coding Optical Setup}

The optical setup is shown in Figure 7. The system is illuminated by a monochromatic spatially incoherent light source; this is achieved by using a HeNe laser and a rotating diffuser. The LCD is located next to the meniscus lens and the ensemble is placed between two linear polarizers to ensure a phase-mostly modulation configuration of the LCD. A transparent object is located in front of the optical system, and finally, a camera is located behind the lens.

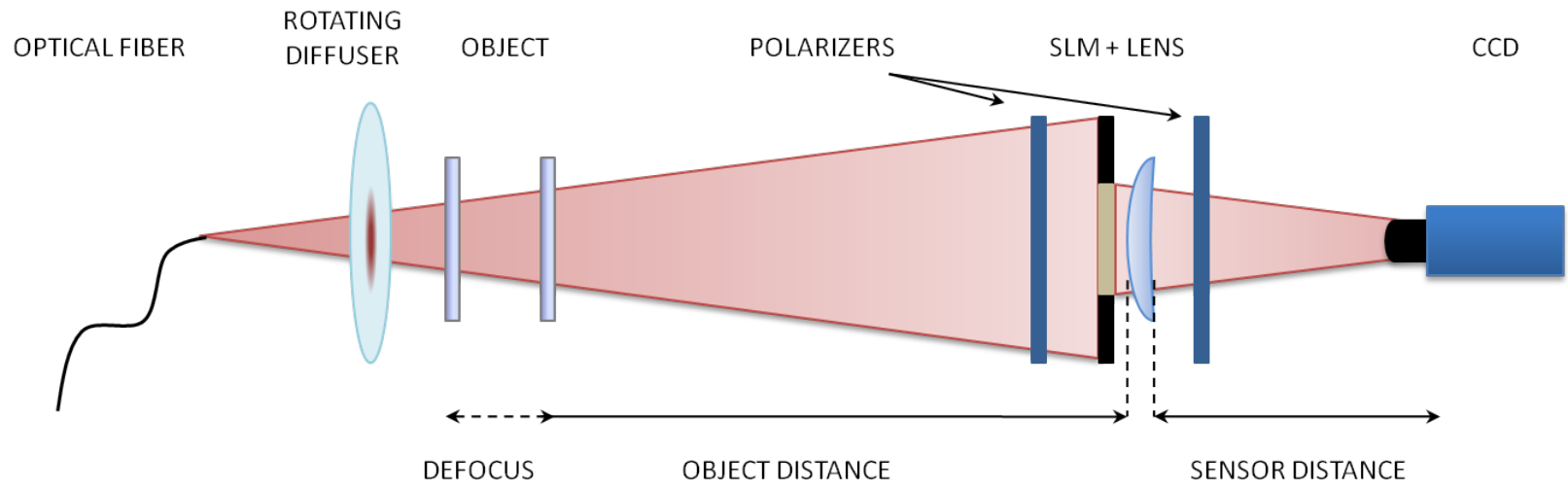

Fig. 7. Optical setup 
A computer simultaneously controls the information displayed on the LCD and the camera that captures the final WFC image. The LCD is linked to the computer by means of the external VGA port, the camera is plugged into a USB or a firewire port. Both devices are controlled by a piece of software written in Labview (wavefrontcoding.vi) using the NI Vision Development Module [23]. This program (see Figure 8) performs several tasks:

1) it loads a pre-calculated PM and displays it on the LCD,

2) it records the output image and displays an average of the frames to minimize noise and

3) it shows the image histogram and a maximum gray level indicator; this feature allows the user to avoid camera saturation by controlling the exposure time; this can be particularly useful when the system PSF is going to be recorded (see below).

\section{E. Wavefront Reconstruction}

The last step involves the deconvolution of the coded image using Equations 9 and 10. The Wiener filter can be used provided that $\left|h_{\mathrm{wfc}}\right|^{2}$ is known. This PSF can be obtained experimentally by removing the rotating diffuser and the scene from the optical setup, as show in Figure 9. Figure 10 shows the experimental PSF obtained. Finally the diffraction-limited image is recovered by using the Wiener filter. This calculation can be carried out using the script wiener.m.

Figure 11 shows several experimental images acquired at different steps in the experiment. The MIL-STD-150A 1951 USAF resolution test chart is used as the input image. Results for the conventional optical system are shown in the first row. Images acquired at $\Delta z= \pm 5 \mathrm{~mm}$ are out-of-focus whereas the image at $\Delta z=0 \mathrm{~mm}$ is focused. In the second row (WFC phase-plate with compensated spherical aberration), the three images are nearly indistinguishable. Finally, the images presented in the third row (Wiener filter reconstructed images) are nearly indistinguishable and their quality has been clearly improved.

\section{Assessment of THE Project}

The lab work presented in this paper was first performed by 28 students as part of the IP course in the spring semester of the 2009-2010 academic year. One year later (spring 2011), the same activity was proposed for the 40 new students who had enrolled on the IP course. The activity was designed as an end-of-course task covering almost all the topics included in the course. 


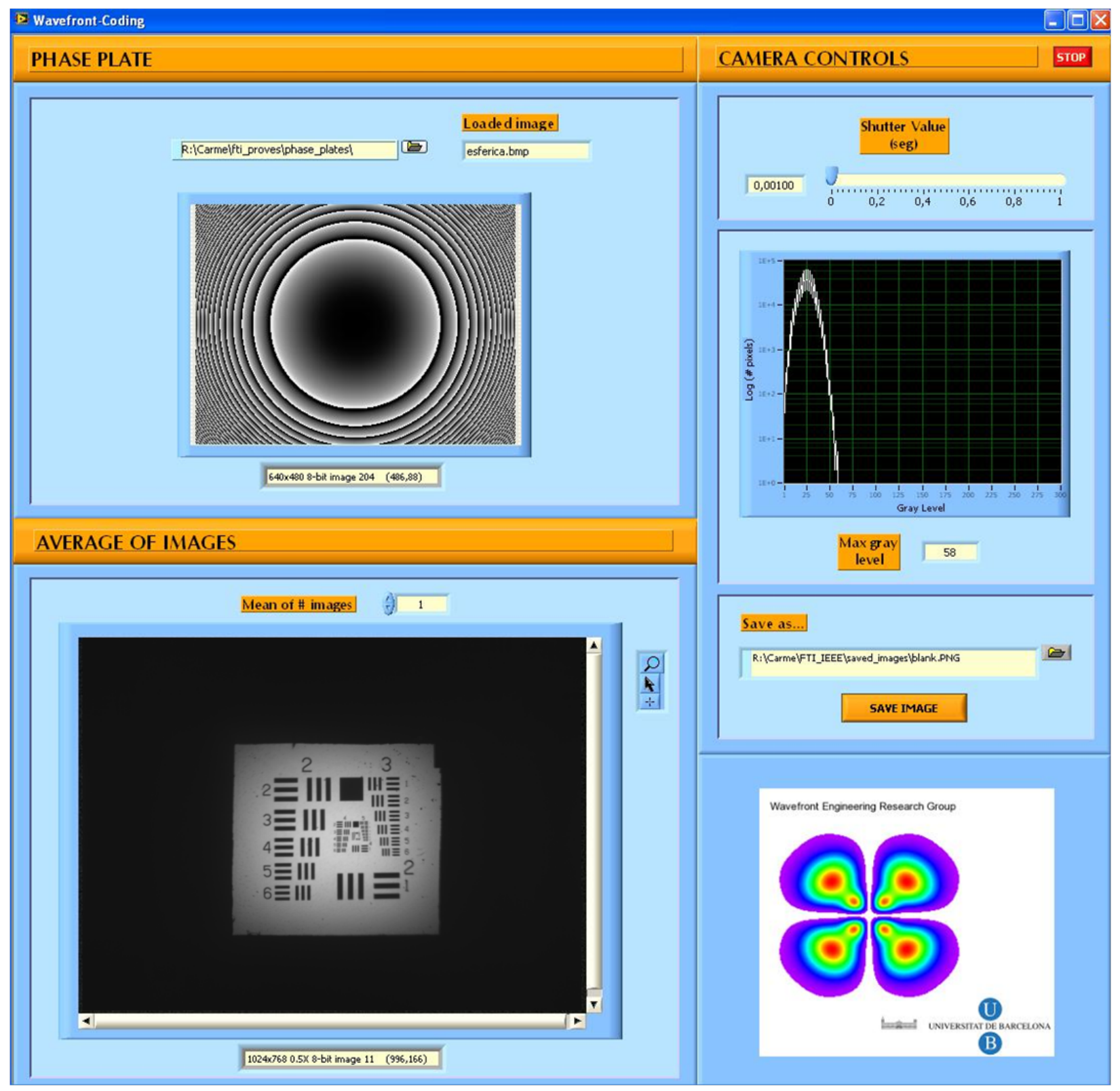

Fig. 8. Main window of the program controlling the LCD and the camera

The experiment is developed over three sessions, with students working in pairs. In the first one-hour session, the instructor presents the topic in the classroom. The second session (two hours) is devoted to preparing the codes to generate the phase mask and calculating the Wiener filter. Due to time limitations, the spherical aberration is calculated by means of a pre-existing Matlab routine. During the third session, the students assemble the optical setup, and carry out 


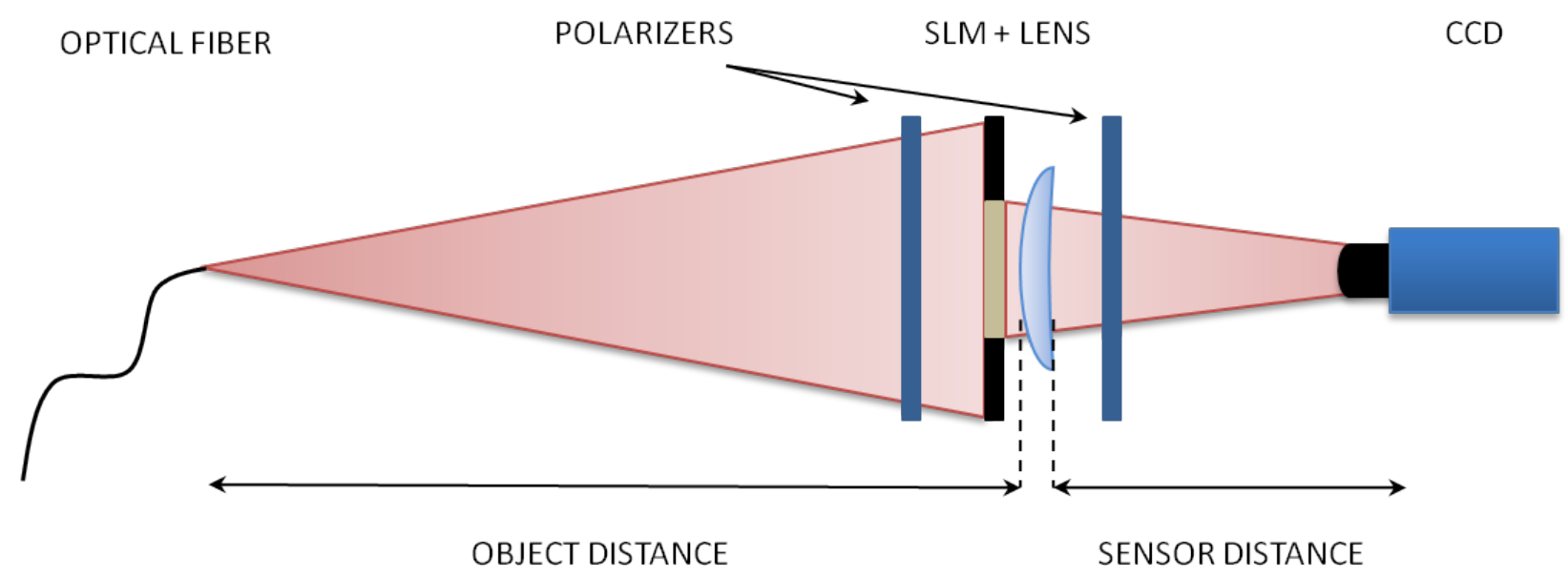

Fig. 9. PSF acquisition.

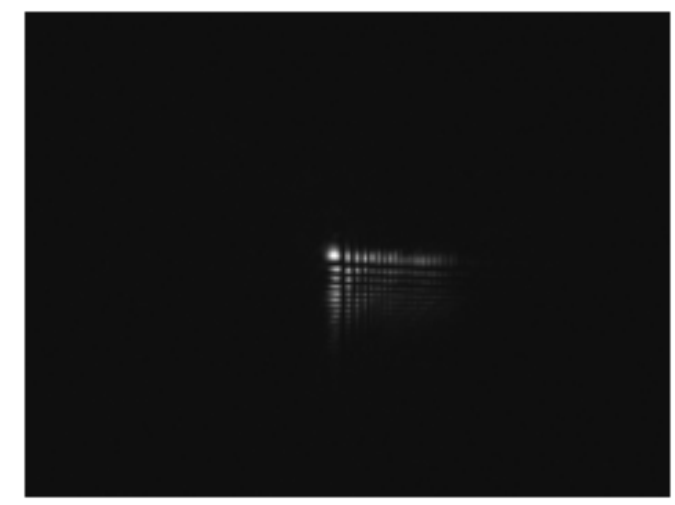

Fig. 10. Experimental coded PSF.

some WFC experiments. Finally, as homework, a final report is required.

\section{A. Spring 2010.}

This lab work was first included in the IP program in 2010. Taking into account its relative complexity, the instructors wanted to know whether the students perceived the experiment as suitable for them. After the students had completed the lab work, they were given a questionnaire designed to survey their perception of it. The questionnaire included a four-item Likert scale and two open-ended questions. Twenty students returned the completed questionnaire. The results are displayed in Table $\mathrm{V}$ and show that the students had a positive opinion of the exercise. 


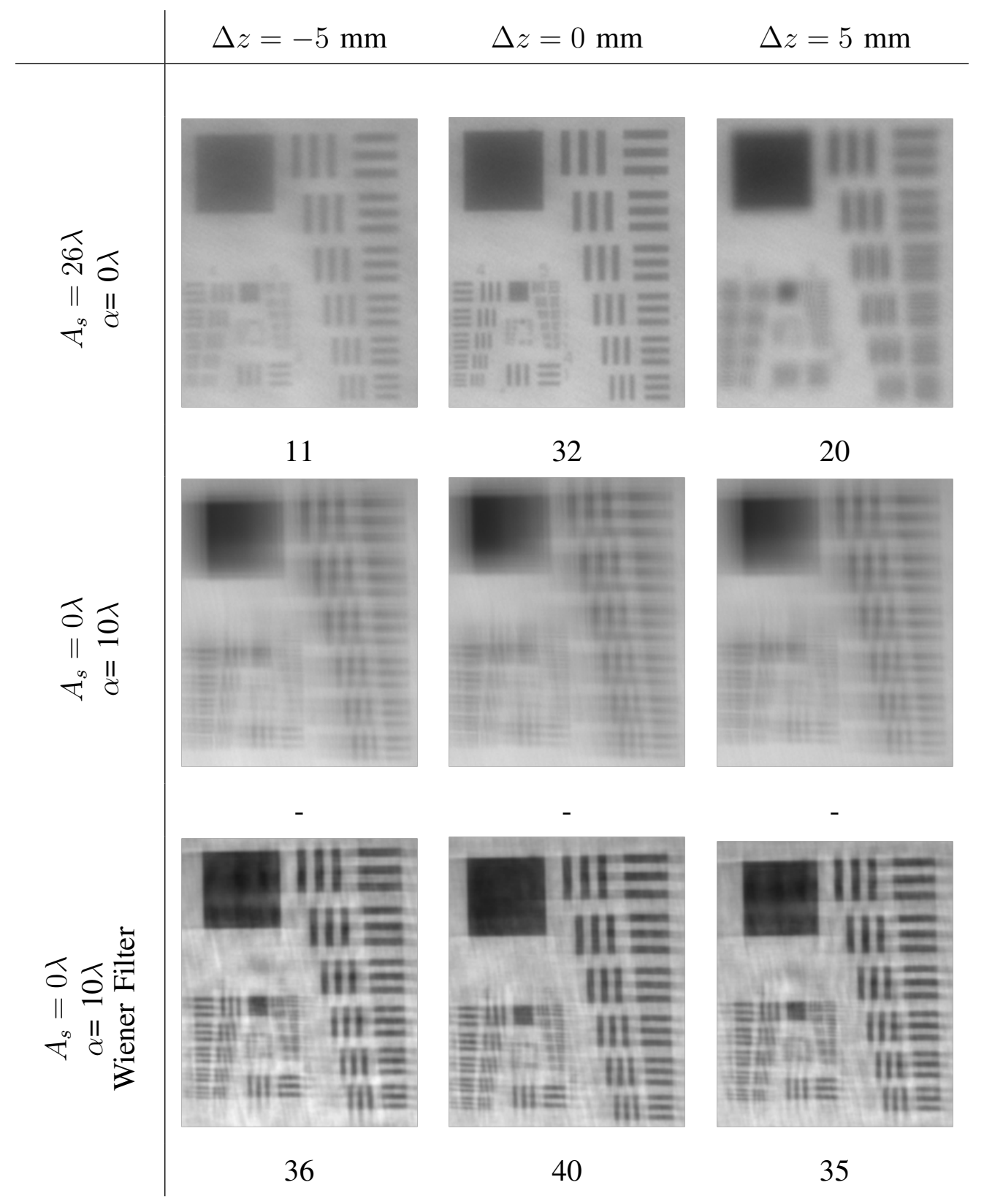

Fig. 11. Experimental image formation process through an optical system. Images saved at $z=0 \mathrm{~mm}, \Delta z=-5 \mathrm{~mm}$ and $\Delta z=5 \mathrm{~mm}$ and the cut-off spatial frequency expressed below in $\mathrm{mm}^{-1}$. First row: conventional optical system. Second row: perfect lens plus cubic phase plate. Last row: Wiener filter reconstructed images.

The two open-ended questions were about the best and the worst aspects of the experience. Students mentioned the following ideas:

- As positive aspects, they noted: that the experiment is up-to-date and linked with the real- 
TABLE V

FREQUENCIES OF THE RESPONSES TO THE ITEMS IN THE LIKERT SCALE FROM THE SPRING 2010 QUESTIONNAIRE. GRAYED CELLS INDICATE THE MOST COMMON RESPONSE TO EACH QUESTION: 1 (DISAGREE), 2 (TEND TO DISAGREE), 3

(TEND TO AGREE) AND 4 (AGREE)

\begin{tabular}{|c|c|c|c|c|c|}
\hline Question & 1 & 2 & 3 & 4 & N/A \\
\hline $\begin{array}{l}\text { The specific training for this practical } \\
\text { activity was enough to succeed in the } \\
\text { task. }\end{array}$ & 1 & 1 & 12 & 6 & 0 \\
\hline $\begin{array}{l}\text { The information included in the cook- } \\
\text { book is appropriate for the preparation } \\
\text { of the activity. }\end{array}$ & 1 & 2 & 12 & 3 & 0 \\
\hline $\begin{array}{l}\text { I have had enough time to prepare the } \\
\text { activity }\end{array}$ & 1 & 3 & 7 & 9 & 0 \\
\hline $\begin{array}{l}\text { I understand the contents of the practi- } \\
\text { cal activity }\end{array}$ & 0 & 4 & 12 & 4 & 0 \\
\hline $\begin{array}{l}\text { The level of the practical activity is } \\
\text { appropriate for this course. }\end{array}$ & 0 & 2 & 10 & 8 & 0 \\
\hline $\begin{array}{l}\text { I would recommend the schedule of } \\
\text { this practical activity for the next year. }\end{array}$ & 0 & 1 & 11 & 8 & 0 \\
\hline
\end{tabular}

world (8 answers); that the project links almost all the topics included in the syllabus (7); and the possibility of working with special equipment (3). Two students highlighted the personalized attention received.

- As for the negative side, the students reported: that they would have preferred to have more time to deal with the setup (9); that some aspects of the guide were not clear enough (3); and that the experiment required an excessive amount of work (3). Five students did not report any negative aspects.

\section{B. Spring 2011}

It has been suggested that the 2011 students answer the same survey that was given to the students who took the course in 2010. About half of the students returned the survey and the results are nearly identical to those obtained in 2010 (Table V). As regards the questions about the best and the worst aspects of the experience, again students mentioned that the lab required the use of most of the topics in the syllabus. They highlighted the fact that the experiment is 
related to current research topics in optical design. On the other hand, some students criticized not having enough time to assemble the optical system and perform the experiment.

In addition to considerations of student perception, how this lab work affects the learning experience of the students was analyzed. Two sources of information were considered:

1) an assessment test composed of 15 multiple choice questions and

2) a lab report, which includes evidence of the experimental results, and the calculations of the phase mask and the reconstruction procedure.

The test and the report where scheduled for two weeks after completion of the lab work. The first seven questions are related to optical system design concepts introduced in section 4 of the IP syllabus: coherent and incoherent PSFs, cut-off frequency and the transfer function, wave aberration, defocus, spherical aberration, etc. Questions 8 to 15 concern concepts connected to the lab work such as the role of certain elements (including the rotating diffuser or the polarizers), how the cubic phase mask affects defocus, how the coded PSF can be acquired, etc. For completeness, the reader can access the English version of the questionnaire at http: //www.ub.edu/waveng/wfc_practical.

Figure 12 shows the number of correct answers to each question. On average, each question was answered correctly 33 times; question 13 (main objective of WFC systems) was answered correctly by all the students (40). The items that caused the most difficulties were related to the transfer function (question 1), the wave aberration of the defocus (question 5), the use of polarizers and the disk diffuser (questions 10 and 14) and the method used to deconvolve coded images (15). This suggests which aspects will require more emphasis in the future.

Figure 13 shows the students grouped according to the number of questions answered correctly: 28 students got good marks (12 to 15 correct answers) whereas 11 made between 4 and 7 mistakes. One student got just 5 answers correct. It is worth pointing out that, on average, 25 students attended the lectures regularly. As expected, these highly motivated students got the best marks.

Two weeks after the end of the lab work the students had to submit a full report comprising: a discussion of how the optical system was assembled; a detailed explanation of how the phase mask and Wiener filter, and their experimental results (including the PSF) were calculated; some coded images in different planes; and the reconstructed Wiener filter images. 30 of the 40 students produced satisfactory work and provided good results, 6 had inaccurate results, and 4 students 


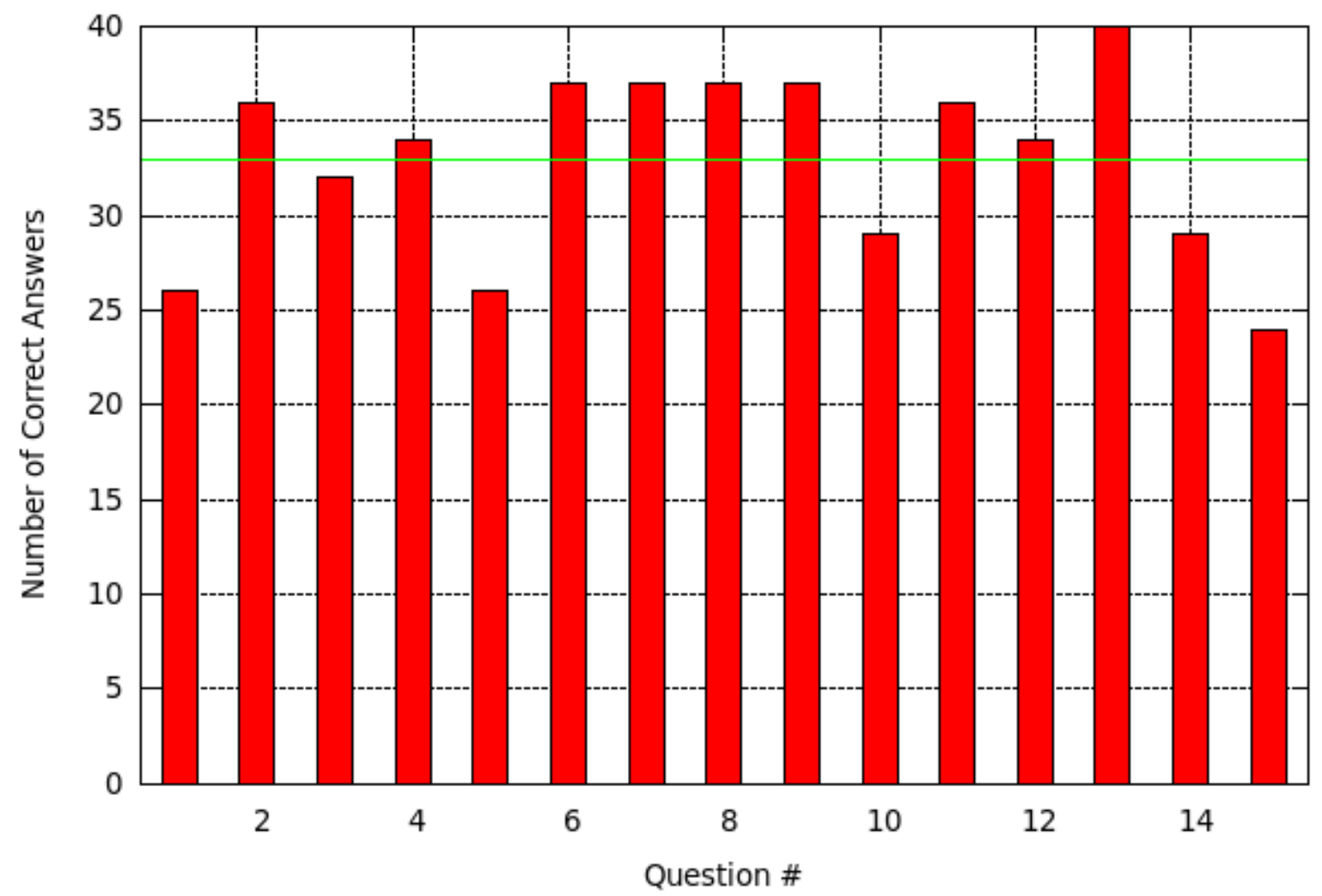

Fig. 12. Number of correct answers to each question. The green line shows the average value.

failed completely. All 26 students who got good results in the test ( 0,1 or 2 mistakes) also succeeded in the lab task.

\section{CONCLUding REMARKS}

This paper reports an academic implementation of a WFC-based optical system using LCDs. The experiment proposed is shown to be a powerful educational tool that requires simultaneous knowledge of several topics and abilities. The activity is suitable for advanced undergraduate students involved in optical engineering related courses. The analysis of the perception of the activity among the students shows that they had a positive opinion of the experience. After performing the lab work, a majority of the students showed suitable theoretical background and a good knowledge of the concepts involved in WFC. 


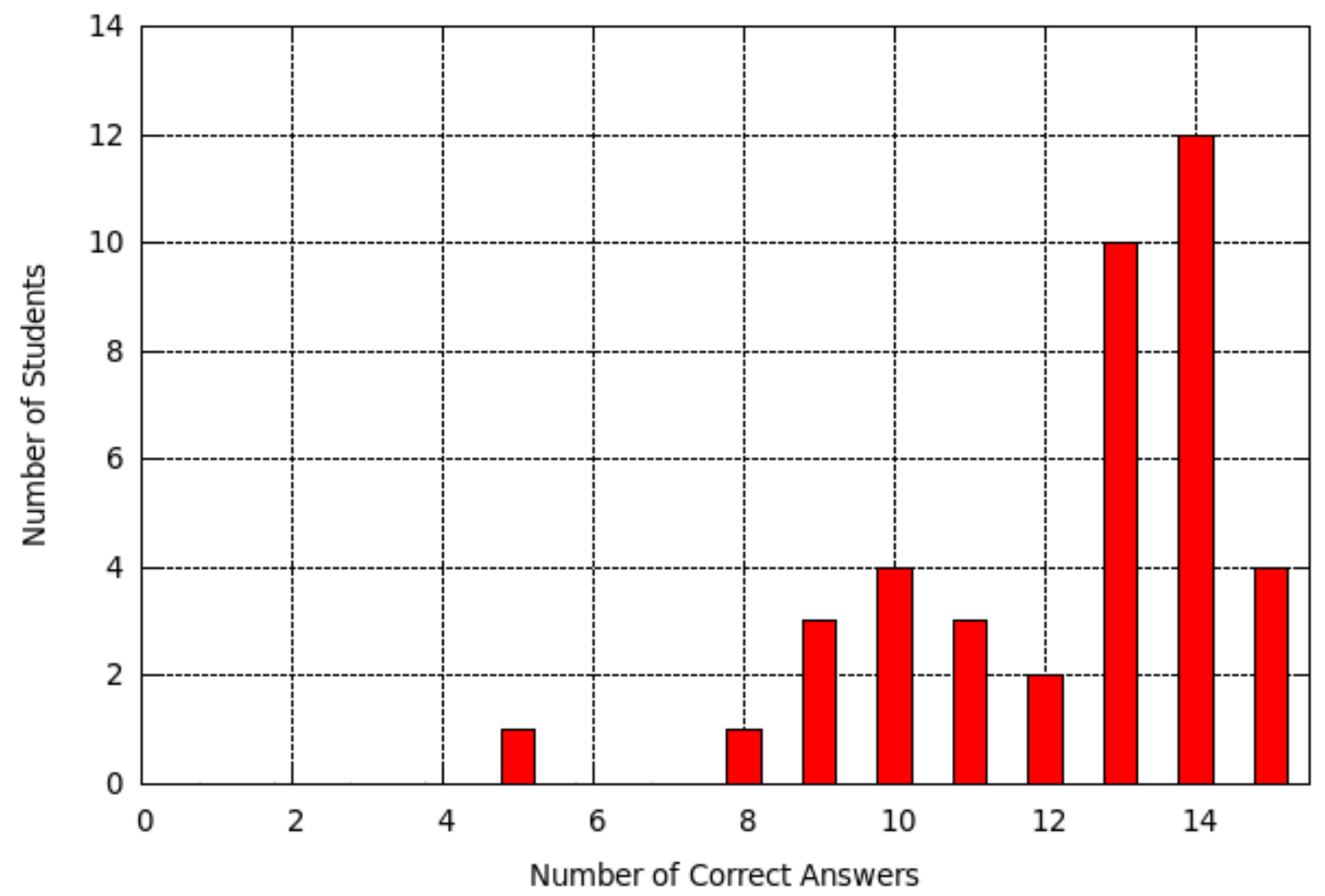

Fig. 13. Number of students giving correct answers.

\section{REFERENCES}

[1] E. R. Dowski and W. T. Cathey, "Extended depth of field through wave-front coding," Applied Optics, vol. 34, no. 11, pp. 1859-1866, 1995.

[2] W. T. Cathey and E. R. Dowski, "New paradigm for imaging systems," Applied Optics, vol. 41, no. 29, pp. 6080-6092, 2002.

[3] R. Van den Berg, "Wavefront coding keeps a focus on applications," Optics \& Laser Europe, 2003, accessed 9-June-2010. [Online]. Available: http://optics.org/cws/article/articles/18342

[4] "Wavefront coding finds increasing use," Laser Focus World, 2004, accessed 9-June-2010. [Online]. Available: http://www.optoiq.com/index/photonics-technologies-applications/lfw-display/lfw-article-display/197348/ articles/laser-focus-world/volume-40/issue-1/world-news/world-news/wavefront-coding-finds-increasing-use.html

[5] A. Harvey and G. Muyo, "Wavefront coding slims down imaging systems," Optics \& Laser Europe, 2007, accessed 9-June-2010. [Online]. Available: http://optics.org/cws/article/research/26632/1

[6] D. Hong, K. Park, H. Cho, and M. Kim, "Flexible depth-of-field imaging system using a spatial light modulator," Applied Optics, vol. 46, no. 36, pp. 8591-8599, 2007.

[7] G. Carles, G. Muyo, S. Bosch, and A. Harvey, "Use of a spatial light modulator as an adaptable phase mask for wavefront coding," Journal of Modern Optics, vol. 57, no. 10, pp. 893-900, 2010. 
[8] R. Cheville, A. McGovern, and K. Bull, "The light applications in science and engineering research collaborative undergraduate laboratory for teaching (LASER Cult)-relevant experiential learning in photonics," IEEE Transactions on Education, vol. 48, no. 2, pp. 254-263, 2005.

[9] B. Anderson, L. Pelz, S. Ringel, B. Clymer, and S. Collins Jr, "Photonics laboratory with emphasis on technical diversity," IEEE Transactions on Education, vol. 41, no. 3, pp. 194-202, 2002.

[10] A. Hermerschmidt, "Experimental modules covering imaging, diffraction, Fourier optics and polarization based on a liquidcrystal cell SLM," in Education and Training in Optics and Photonics. OSA Technical Digest Series. Optical Society of America, 2009.

[11] G. Johnson, E. Dowski, and W. Cathey, "Passive ranging through wave-front coding: information and application," Applied Optics, vol. 39, no. 11, pp. 1700-1710, 2000.

[12] E. Hecht, Optics. Adisson-Wesley, 2001.

[13] J. Goodman, Introduction to Fourier Optics. McGraw-Hill, 1996.

[14] V. N. Mahajan, Optical Imaging and Aberrations, Part II. Wave Diffraction Optics. SPIE Press Books, 2001.

[15] M. Born and E. Wolf, Principles of Optics. Cambridge University Press, 1999.

[16] S. Prasad, T. C. Torgersen, V. P. Pauca, R. J. Plemmons, and J. van der Gracht, "High-resolution imaging using integrated optical systems," International Journal of Imaging Systems and Technology, vol. 14, no. 2, pp. 67-74, 2004.

[17] R. C. Gonzalez and R. E. Wood, Digital Image Processing. Pearson Education, 2008.

[18] S. Sherif and T. Cathey, "Depth of field control in incoherent hybrid imaging systems," in Optical Imaging and Microscopy, ser. Springer Series in Optical Sciences, P. Török and F. Kao, Eds., vol. 87. Springer, 2007, pp. 137-168.

[19] “OptiXplorer - Optics Education Kit," accessed 31-May-2011. [Online]. Available: http://www.holoeye.com/optics_ education_kit_optixplorer.html

[20] I. Labastida, A. Carnicer, E. Martin-Badosa, S. Vallmitjana, and I. Juvells, "Optical correlation by use of partial phase-only modulation with VGA liquid-crystal displays," Applied Optics, vol. 39, no. 5, pp. 766-769, 2000.

[21] A. Márquez, C. Iemmi, I. Moreno, J. Campos, and M. Yzuel, "Anamorphic and spatial frequency dependent phase modulation on liquid crystal displays. Optimization of the modulation diffraction efficiency," Optics Express, vol. 13, no. 6, pp. 2111-2119, 2005.

[22] W. Welford, Aberrations of optical systems. Taylor \& Francis, 1986.

[23] “NI Vision Development Module," accessed 9-June-2010. [Online]. Available: http://www.ni.com/vision/vdm.htm

\section{ACKNOWLEDGMENT}

We thank Dr. Guillem Carles and professors Santiago Vallmitjana and Jordi Cuadros for their helpful comments. The authors are indebted to our students for their feedback and suggestions

Carme Ferran (Barcelona, 1981) was awarded her Bs.C. degree in Physics in 2007 from the Autonomous University of Barcelona (UAB) and her M.Sc. degree in Engineering Physics in 2009 from the Universitat the Barcelona (UB). 
Salvador Bosch (Reus, 1957) received his B.Sc. and Ph.D. degrees in Physics in 1980 and 1985 from the University of Barcelona. His research topics have mainly been image quality and processing, thin film optics and diffractive optics. Currently he is Professor in Optics at the Applied Physics and Optics Department of the University of Barcelona.

Artur Carnicer (Barcelona, 1965) received his B.Sc. and Ph.D. degrees in Physics in 1989 and 1993 from the UB.

Currently, he is an Associate Professor in the Applied Physics and Optics Department at the UB where he teaches undergraduate and graduate courses in physical optics and photonics. His research focuses on the development of computational methods for the study of non-paraxial electromagnetic fields.

Prof. Carnicer is member of the Catalan Physical Society and the Royal Spanish Physical Society. 